\title{
XVII. Ueber den Apparat zur Untersuchung und Brechung des Lichtes von Ibn al Hattam; von Etihard Wiedemann.
}

Von den ältesten Instrumenten, die zur Bestimmung der Brechung im Wasser benutzt wurden, sind uns ron denjenigen von Ptolemäus und Al Hazen (Ibnal Haitam) Beschreibungen erhalten; dieselben unterscheiden sich voneinander etwas, wenn auch nicht wesentlich. Von dem Apparat des Ibn al Haitam enthält der schon mehrfach citirte Codex in Leyden Abbildungen, die ich mir mitzutheilen erlaube. Die erste Figur gibt die Vorder-, die zweite die Hinteransicht.

Die Erklärung der Figur ergibt sich aus der Beschreibung des Instrumentes, wie ich dieselbe in etwas gekürzter Form nach der lateinischen Uebersetzung von $R$ isner ${ }^{1}$ ) wiedergebe, der arabische Text stimmt vollkommen mit derselben überein. Bei der Zeichnung ist zu beachten, dass die ausserhalb des Kreises mit dem Durchmesser ${ }_{C}$ gelegenen Theile senkrecht auf der Ebene der Zeichnung stehen, ebenso wie das Trapez bei $e$ mit der Oeffnung $d$.

Die Beschreibung lautet:

Man nimmt eine runde, ziemlich starke Scheibe aus Kupfer von wenigstens einer Elle Durchmesser. Sie muss einen Rand haben, der senkrecht auf ihrer Oberfläche stebt und wenigstens drei Finger breit ist. In der Mitte des Rückens der Scheibe muss sich eine kleine runde Säule (Fig. $2 b$ ) von wenigstens drei Finger Länge befinden, die senkrecht auf der Oberfläche der Scheibe steht.

(In der Figur ist die Scheibe der innere Kreis, der Rand der an diesen sich nach aussen anschliessende Theil.)

Dieses Instrument befestigen wir so auf der Drehbank, auf der die Drechsler ihre Kupfergeräthe drehen, dass die eine Spitze derselben auf die Mitte der Scheibe, die andere auf die Mitte der kleinen Säule kommt, und drehen den Apparat so lange $a b$, bis die Ränder innen und aussen

1) Opticae thesaurus Al Hazen. Arabis libri septum nunc primum edita a. F. Risnero, Basileae 1572. p. 231-233. 
vollständig kreisrund und glatt sind, und. die kleine Säule auch kreisrund ist. Hierauf ziehen wir auf der inneren Oberfläche des Instrumentes zwei aufeinander senkrechte Durchmesser, dann bezeichnen wir einen Punkt auf der Basis des Randes des Instrumentes, dessen Abstand rom Ende eines der beiden Durchmesser eine Fingerbreite beträgt. Von diesem Punkte aus ziehen wir einen dritten

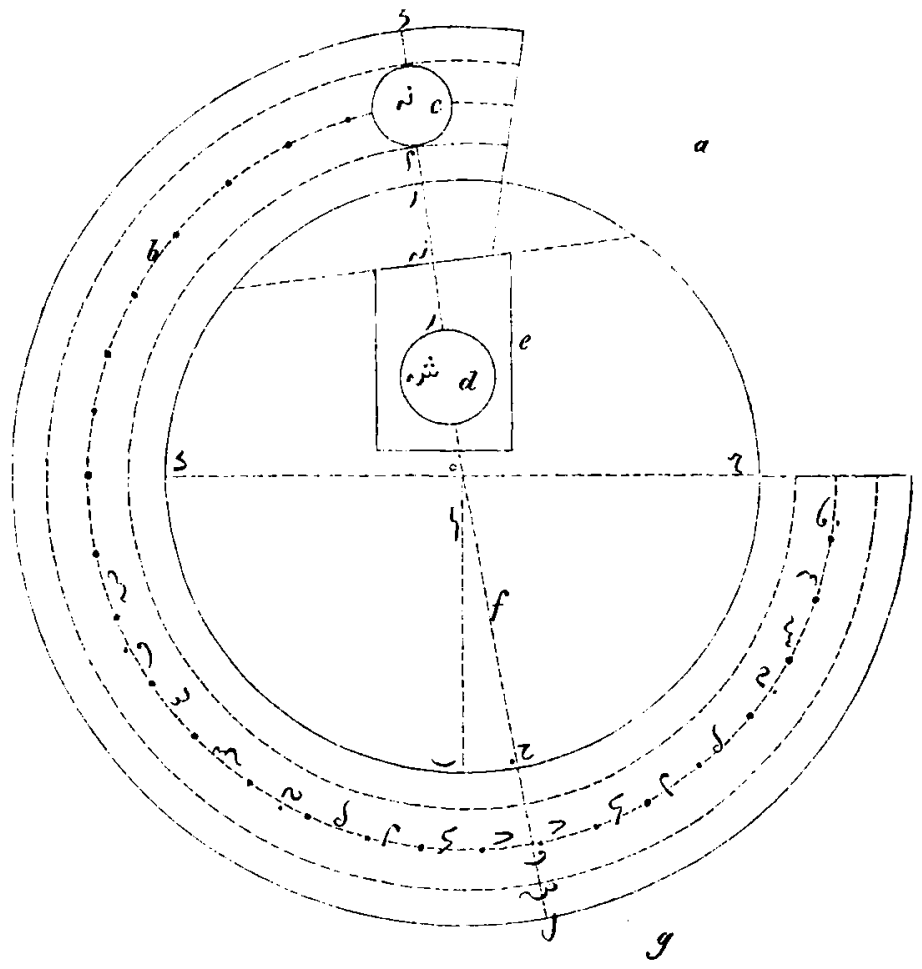

Fig. 1.

Durchmesser durch die Mitte der Scheibe. Dann ziehen wir von den beiden Enden dieses Durchmessers aus zwei Linien auf dem Rande, senkrecht zur Oberfläche der Scheibe. Auf der einen dieser beiden Linien bezeichnen wir von der Scheibe aus drei etwa um die Länge eines halben Gerstenkornes voneinander abstehende Punkte und ziehen auf der 
Drehbank duroh diese Punkte drei voneinander gleichweit abstehende Kreise, die natürlich die gegenüberliegende kurze Linie gleichfalls in drei gleichweit voneinander abstehenden Punkten schneiden. Dann theilt man den mittleren Kreis in 360 Grade und womöglich noch in Minuten. In den Rand bohrt man ein kreisförmiges Loch, dessen Mittelpunkt der mittlere der obigen drei Punkte ist, und dessen Durchmesser gleich dem Abstand der beiden äussersten ist. Nun nehmen wir ein mässig dünnes, genau rechteckiges ebenes Stück Blech $d$ von der Höhe des Randes und etwa gleicher Breite. Von der Mitte der einen Seite ziehen wir eine zu

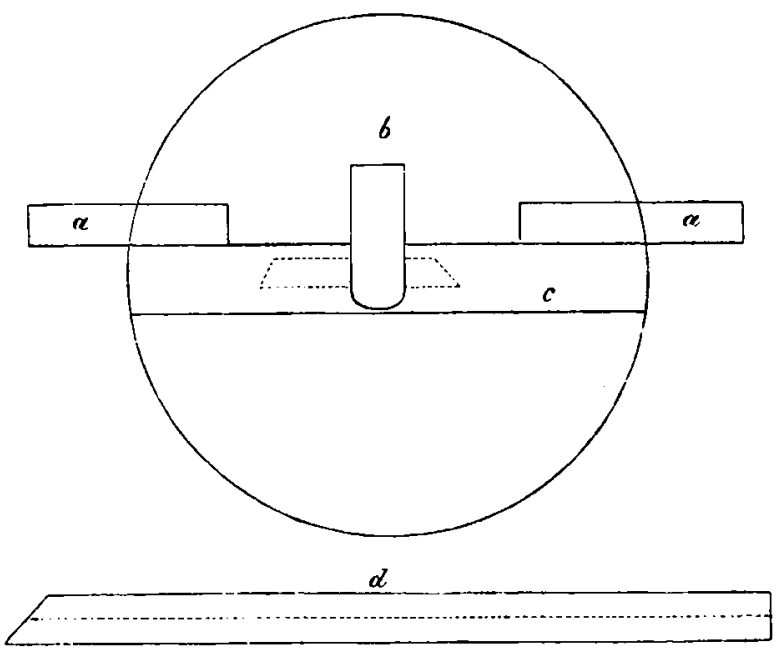

Fig. 2.

dieser senkrechte Linie, auf der wir drei Punkte, die gleichweit voneinander abstehen, bezeichnen. Ihr Abstand a sei dabei gleich den Abständen je zweier der Kreise auf dem Rande. Wir bohren dann in die Platte ein rundes Loch, dessen Mittelpunkt dem mittleren der obigen Punkte entspricht, und dessen Radius gleich dem Abstande $a$ ist. Wir erhalten so ein Loch, das vollkommen mit dem im Rande des Instrumentes correspondirt. Darauf sucht man den 
Mittelpunkt des Radius, welcher den Mittelpunkt der Scheibe mit der Linie auf dem Rande verbindet, auf welcher sich das Loch befindet, und zieht durch ihn eine Senkrechte zu dem Radius; längs dieses befestigt man nun vollkommen fest das kleine Blech, sodass die Mitte desselben genau auf den Radius zu liegen kommt, die kleine Oeffnung in ihr liegt dann genau derjenigen auf dem Rande gegenüber. Die Verbindungslinie der Mittelpunkte der beiden Oeftnungen liegt in der Ebene des mittleren der beiden Kreise auf dem Rand, liegt parallel zu dem Durchmesser auf der Scheibe und verhält sich wie die Absehe beim Astrolab. Hierauf schneidet man aus dem Rande des Instrumentes dasjenige Viertel aus, welches an das Viertel sich anschliesst, in welchem sich das Loch befindet, und welches durch die zwei ersten Durchmesser bestimmt ist, und gleicht den Rand genau ab. Hierauf nimmt man ein quadratisches Stück Metall von eher mehr als einer Elle Länge und feilt die Flächen desselben möglichst senkrecht zu einander ab. In der Mitte derselben bohrt man ein Loch senkrecht zu der einen Fläche, sodass sich der obenerwähnte säulenförmige Theil schwierig in demselben drehen lässt. In dieses Loch setzt man den säulenförmigen Theil ein. Von dem Metallstück schneidet man soviel ab, dass es gleich steht mit dem Rande der Scheibe, und legt die beschnittenen Enden auf die Enden des Metallstückes und verbindet sie mit denselben. ${ }^{1}$ ) Zweckmässig ist es, durch das Ende der kleinen Säule, die aus der Oeffnung im quadratischen Stück hervorragt, einen kleinen Stift zu treiben.

Die Messungen werden so angestellt, dass man das Instrument bis zum Mittelpunkt ins Wasser taucht, der Verbindungslinie der beiden Oeffnungen verschiedene Neigungen gegen den Horizont gibt und den Mittelpunkt des Bildes unter dem Wasser bestimmt, wenn die Sonnenstrahlen eben die beiden Oeffnungen durchsetzen.

1) Dies dient dazu, damit man beim Einsenken des Apparates in ein Gefäss voll Wasser das Nivean desselben mit cinem Durchmesser zusammenfallen lassen kann. 